\section{PERIFERIZAÇÃO DAS PRÁTICAS INTEGRATIVAS E COMPLEMENTARES NA ATENÇÃO PRIMÁRIA À SAÚDE: DESAFIOS DA IMPLANTAÇÃO DO LIAN GONG COMO PRÁTICA DE PROMOÇÃO À SAÚDE}

\author{
Peripheralization of Integrative and Complementary Practices \\ at Primary Health Care: The challenge of Lian Gong as a \\ practices of Health Promotion
}

Artigo Original

\title{
RESUMO
}

Objetivo: Identificar os principais obstáculos enfrentados na implantação do programa Lian Gong em 18 Terapias (LG18T) na Atenção Primária à Saúde (APS) em Belo Horizonte, Brasil. Métodos: Estudo qualitativo feito com 6 grupos focais com instrutores de Lian Gong (2013), 5 grupos focais com Gerentes de Centros de Saúde de Belo Horizonte (2014) e 3 grupos focais com praticantes de LG18T (2015). Após transcrição e análises, resultou na seguinte categoria temática: Periferização das Práticas Integrativas no Modelo Assistencial. Resultados: Falta de apoio real dos gestores e Equipes de Saúde da Família no desenvolvimento das ações de Promoção da Saúde na APS; A visão institucional ainda é focada na produção e na pressão pelos atendimentos assistenciais aos usuários, em detrimento às ações de Promoção da Saúde. Conclusão: Existe uma grande necessidade de disseminar as Práticas Corporais e de Promoção da Saúde no contexto da APS.

Descritores: Promoção da Saúde; Atenção Primária à Saúde; Medicina Tradicional Chinesa.

\section{ABSTRACT}

Objective: To identify the main obstacles faced in the implementation of Lian Gong program in 18 Therapies (LG18T) in Primary Health Care (PHC) in Belo Horizonte, Brazil. Methods: Qualitative research with six focus groups with Lian Gong instructors (2013), 5 focus groups with health centers Managers of Belo Horizonte (2014) and 3 focus groups with practitioners LG18T (2015). After transcription and analysis resulted in the following thematic categories: Institutional Care Model and Culture. Results: There is a lack of real suport of managers and Family Health Teams in the development of health promotion actions in PHC; The institutional view is still focused on production and pressure by assistance calls to users over the health promotion actions. Conclusion: There is a need to disseminate the effectiveness of Body Practices and Health Promotion in PHC.

Descriptors: Health Promotion; Primary Health Care; Chinese Traditional Medicine.

1) Universidade Federal de Minas Gerais UFMG - Belo Horizonte (MG) - Brasil

2) Programa de Lian Gong na Prefeitura de Belo Horizonte - Belo Horizonte (MG) - Brasil

Este artigo seguiu as normas e formatação estabelecidas pelo $5^{\circ}$ CIAIQ - Congresso Ibero-Americano em Investigação Qualitativa. 


\section{INTRODUÇÃO}

A importância das práticas de Promoção da Saúde e das Práticas Integrativas e Complementares (PIC), no Brasil, começou a ser discutida na $8^{\mathrm{a}}$ Conferência Nacional de Saúde em 1986 quando foi assumido o conceito ampliado de saúde, o qual busca superar a ideia de saúde como ausência de doença. Ambas ganham importância em 2006, com a criação da Política Nacional de Práticas Integrativas e Complementares (PNPIC) e Política Nacional de Promoção da Saúde (PNPS), em revisão realizada em 2014, reforça o conceito de promoção da saúde e das práticas que visam melhorias da qualidade de vida.

Além disso, a Promoção da Saúde deve articular dois elementos da concepção de saúde que combina ações, desenvolvidas de forma conjunta por diferentes setores do governo e da sociedade civil, para a melhoria das condições de vida e saúde, promovendo a equidade e o desenvolvimento da cidadania ${ }^{(1)}$. Nesse sentido, incluem-se as práticas integrativas.

As Práticas Integrativas e Complementares (PIC) são técnicas e saberes do cuidar de si e dos outros, que atuam com uma atenção humanizada, visando o princípio da integralidade, reequilíbrio por mecanismos naturais e de baixo risco. No Brasil, as PIC são regulamentadas pela Política Nacional de Práticas Integrativas e Complementares, que busca incentivar formas diferenciadas de produzir saúde e de melhorar a qualidade de vida da população, por acreditar que existe uma adesão e participação ativa da população nestas práticas, além de baixo custo para treinar profissionais da saúde ${ }^{(2)}$.

A Organização Mundial de Saúde, por meio do documento Traditional Medicine Estrategy 2014-2023, discute as PIC no sentido de auxílio no desenvolvimento de políticas nacionais, por acreditar na real contribuição delas para a saúde no mundo e para os sistemas de saúde de cada nação em modo particular. Neste documento, também foram listadas as principais dificuldades enfrentadas pelos Estados na implementação das práticas, a saber: a falta de dados provenientes de pesquisas científicas; a falta de conhecimentos especializado entre as autoridades sanitárias e os órgãos competentes; a dificuldade de estabelecer indicadores do desempenho e de assim, avaliar a qualidade e eficácia da prática; a falta de profissionais capacitados para atuar nas $\mathrm{PIC}^{(3)}$.

Algumas PIC estão vinculadas à Medicina Tradicional Chinesa, uma tradição milenar proveniente do Oriente que adota uma postura vitalista diante do corpo, da saúde e da doença como um estado geral. Uma nova forma de pensamento que não está em busca da "cura" de doenças ou de ver o corpo como um agrupamento de órgãos e tecidos com funções fisiológicas ${ }^{(4)}$, que possuem meios fortalecedores do autocuidado e da busca pela reconstrução das relações do sujeito consigo e com o seu meio natural e social ${ }^{(5)}$. A ênfase da MTC é no processo saúde-doença, usada como alternativa aos tratamentos da medicina convencional. A MTC é uma complexa interação entre tradição chinesa e modernidade, e a doença é vista como um desequilíbrio interno, com causas profundas nos indivíduos e no seu modo de viver ${ }^{(6)}$. No Brasil existem algumas modalidades de MTC difundidas, como a acupuntura, Tai-Chi-Chuan, Lian Gong em 18 Terapias, Qigong, entre outras.

O Lian Gong em 18 terapias (LG18T) foi desenvolvido no ano de 1974, durante o êxodo rural ocorrido na China, retratado pela transição dos trabalhadores do campo para as cidades. Com isso, ocorreu o aumento nas doenças decorrentes do trabalho nas fábricas, além de queixas como as dores no corpo. Motivado por este cenário, o Dr.Zhuang Yuan Ming, médico ortopedista, criou as 18 terapias de Lian Gong. A prática é composta por movimentos suaves e firmes para as regiões do corpo como pescoço, ombros, costas, região lombar e membros inferiores realizadas por pessoas de qualquer idade, como um recurso terapêutico de auto prática capaz de intervir na saúde dos praticantes ${ }^{(7)}$. Os benefícios da prática corporal chinesa transcendem o objetivo de redução de dores, e atuam também na melhoria da qualidade do sono, redução do uso de medicamentos e socialização, além da redução na busca por serviços de saúde $^{(8)}$. O Lian Gong em 18 terapias como uma prática de Promoção da Saúde vem trazendo muitos ganhos para a qualidade de vida e relações sociais dos praticantes, além da redução na medicalização (principalmente nos medicamentos de indução do sono, dor e depressão) e na procura pelo acolhimento nos centros de saúde. Em Belo Horizonte, o Lian Gong em 18 Terapias é ofertado na APS em 222 locais, totalizando cerca de 11.500 praticantes em todo o município ${ }^{(9)}$.

Embora as PIC e as práticas de Promoção da Saúde, de um modo geral apresentam substancial crescimento no Brasil e no mundo, a sua institucionalização na APS ainda está em processo de consolidação. Para existir a valorização dos novos procedimentos nos serviços de saúde seria preciso haver uma validação, por meio de evidência científica, da sua eficácia. O que no momento, apresenta como ponto frágil, em relação às práticas. Portanto, acredita-se que muitos desafios ainda prevalecem, para a sua efetivação nos serviços de saúde ${ }^{(10)}$.

Nesse sentido, traçou-se como objetivo deste estudo, identificar os desafios enfrentados na implantação de programas de Promoção da Saúde, investigando o programa de Lian Gong em 18 terapias na Atenção Primária do município de Belo Horizonte, Minas Gerais, Brasil. 


\section{MÉTODOS}

O estudo utilizou-se de metodologia qualitativa por meio da realização de grupos focais, com o objetivo de pontuar quais as principais dificuldades e barreiras enfrentadas por gerentes de unidades de saúde, instrutores e praticantes de Lian Gong acerca da implantação e adesão da prática na atenção à saúde. Segundo Minayo, os grupos focais são um dos modelos de entrevista ou conversa em grupos pequenos e homogêneos.

"Visam obter informações, aprofundando a interação entre os participantes, seja para gerar consenso, seja para explicitar divergências [...] Por isso são usados para focalizar a pesquisa e formular questões mais precisas; complementar informações sobre conhecimentos peculiares a um grupo em relação a crenças, atitudes e percepções; desenvolver hipóteses para estudos complementares; e como técnica exclusiva"(II).

Foi realizado o total de 14 grupos focais, sendo $06 \mathrm{com}$ instrutores de LG18T convidados a participar durante as aulas de manutenções realizadas no Instituto Mineiro de Tai Chi e Cultura Oriental, 05 grupos focais com os gerentes dos Centros de Saúde que ofertam a prática de Lian Gong e $03 \mathrm{com}$ os praticantes de LG18T do município de Belo Horizonte.

Após o preenchimento do Termo de Consentimento Livre e Esclarecido (TCLE), os participantes foram estimulados a falar e refletir sobre a prática de Lian Gong em sua realidade a partir de questões norteadoras desenvolvidas em parceria com a coordenação municipal do programa de Lian Gong.

O material dos grupos focais realizados foi transcrito em seguida, realizado leitura flutuante, a fim favorecer a identificação de categorias temáticas. A partir das categorias foram organizados e analisados os conteúdos por meio da Técnica de Análise de Conteúdo de Bardin resultando na categoria Periferização das Práticas Integrativas no Modelo Assistencial $^{(12)}$.

O presente estudo constitui um recorte da pesquisa intitulada "Avaliação da Efetividade do Programa de Lian Gong em 18 Terapias na Atenção Primária à Saúde do município de Belo Horizonte, Minas Gerais" que está sendo desenvolvida na Universidade Federal de Minas Gerais pelo Curso de Gestão de Serviços de Saúde, aprovada pelo comitê de ética, projeto: CAAE- 14055613.0.0000.5149.

\section{RESULTADOS E DISCUSSÃO}

Para os Orientais adeptos à Medicina Tradicional Chinesa (MTC), culturalmente não existe a tradição de avaliar cientificamente a efetividade das práticas corporais. No entanto, para os ocidentais essa postura é incompreensível. Com a inserção das práticas corporais nos países ocidentais, é premente a necessidade de estudos que confirmam seus benefícios, e que comprovam a sustentabilidade do programa como uma política pública ${ }^{(13)}$. Diante disso, o estudo em questão identificou os principais desafios na implantação do Lian Gong em 18 Terapias em Belo Horizonte.

\section{Periferização das Práticas Integrativas no Modelo Assistencial}

A categoria temática, periferização das Práticas Integrativas no Modelo Assistencial, foi evidenciada por aspectos relacionados a hegemonia do modelo biomédico, falta de apoio institucional, espaços adequados, divulgação incipiente para o desenvolvimento das práticas corporais, por parte das gerências locais e equipes de saúde.

A hegemonia do modelo biomédico alimentou, ao longo da história, uma cultura de valorização da doença alimentando uma cultura institucional, dos Centros de Saúde, que desvaloriza as ações de promoção da saúde, constituindo-se como um dos principais desafios enfrentados pelas práticas. Esta cultura se revela em ações como a falta de apoio real dos gestores de Centros de Saúde e equipes de saúde da família no desenvolvimento das ações de promoção da saúde na Atenção Primária à Saúde, e no produtivismo em saúde, com a priorização de consultas individualizadas contra as ações coletiva, ilustrada com a fala de um dos instrutores: "[...] dependendo do momento você recebe uma pressão, às vezes é sutil, olha estamos precisando do seu horário para atendimentos, dos horários para outras coisas." (GF, instrutores).

A fala acima ressalta a falta de apoio dos gerentes e o número insuficiente de profissionais da saúde que acreditam no desenvolvimento das atividades de Promoção da Saúde e são considerados pelos instrutores uma barreira para o programa de práticas corporais Lian Gong.

Reforça-se que quando o alcance de metas e indicadores curativistas são priorizados pelo gestor de saúde e profissionais envolvidos, fica mais difícil se desenvolver ações que busquem promover a saúde das populações e se torna claro o papel de apoio institucional do gestor no processo de trabalho em saúde ${ }^{(14)}$.

A visão institucional ainda é focada na produção e na pressão pelos atendimentos assistenciais aos usuários dentro dos Centros de Saúde, em detrimento às ações de Promoção da Saúde que, muitas vezes não são priorizadas ${ }^{(15)}$. Como no caso do Lian Gong, onde a prática não é considerada uma atividade de rotina como quaisquer outras práticas assistencialistas desenvolvidas na atenção primária, podendo ser visto nas seguintes falas: 
"Aqui é seu horário de trabalho e você tem que estar aqui” (GF, INSTRUTORES).

"Muitas vezes eles são estimulados a fazer, eles querem fazer, mas depende da escala, eu não posso deixar um horário descoberto e eles mesmo tem essa consciência" (GF, GERENTES).

“Mas[...] olha, manutenção não vai dar para te liberar, [...] não dá porque tem reunião com as equipes de PSF e não sei o que é no mesmo horário” (GF INSTRUTORES).

A cultura institucional está enraizada no modelo biomédico, que não entende que as ações de promoção são atividades importantes e necessárias, também, para os profissionais que executam as práticas, isso é uma barreira para os programas de promoção.

Outro desafio enfrentado é a falta de espaços adequados para a realização da prática. Sendo que na Política Nacional de Promoção da Saúde ${ }^{(16)}$ é preconizado que para a implementação e continuidade das práticas corporais é preciso uma área física adequada, equipamentos necessários e uma equipe capacitada. Durante o grupo focal realizado com os gerentes das unidades de saúde e com os instrutores de Lian Gong esta foi uma dificuldade ressaltada diversas vezes como nas falas a seguir:

"Eu acho que é esse o dificultador a gente ficar procurando espaço pra poder colocar em prática, as unidades quase nenhuma tem espaço que possibilite isso. (GF, GERENTES).

"Eu tenho mais de setenta alunos, agora não vão todos porque a sala não cabe [...]” (GF, INSTRUTORES).

As dificuldades em identificar locais adequados para a prática faz com que muitos instrutores tenham um número restrito de praticantes, influenciado por problemas relacionados ao espaço da prática como por exemplo, utilização de sala de reuniões ou estacionamento da unidade de saúde.

Embora exista uma valorização das PIC no SUS é evidente a sua periferização institucional e dentre os possíveis motivos estão o fato de ser pouco percebida pelos profissionais de saúde e pelos grupos sociais e por tanto, a população de um modo geral valorizar pouco este tipo de prática de saúde ${ }^{(5)}$.

A falta de espaço físico e apoio logístico também representaram uma barreira na introdução do Lian Gong na Unidade Básica de Saúde Villalobo na cidade de São Paulo em 2002, se tornando necessário fazer parcerias com a comunidade local, desenvolvendo a prática em escolas, igrejas, associações e no asilo ${ }^{(17)}$. Estes estudos reforçam que a falta de infraestrutura representa uma barreira para o desenvolvimento das práticas corporais como práticas de Promoção da Saúde.
Outro desafio enfrentado pelo Lian Gong em Belo Horizonte é que uma parte dos instrutores que receberam a especialização, ofertada pela prefeitura, deixam de oferecer a prática por diversos motivos. Dados apresentados pela coordenação municipal do programa de Lian Gong em 18 Terapias da prefeitura de Belo Horizonte demonstram que, em 2015, dos 456 instrutores capacitados, apenas 303 oferecem a prática regularmente no SUS e alguns motivos citados são a falta de apoio institucional, de espaço para a realização da prática e de horário na agenda do profissional.

Apesar de o Lian Gong estar inserido na Atenção Primária à Saúde, a divulgação nos Centros Saúde por meio de cartazes, indicação dos profissionais de saúde, dos gerentes e dos estudantes que participam do programa "Posso Ajudar" ainda é pouco expressiva. Em algumas falas dos gerentes de Centros de Saúde que ofertam a prática corporal esta barreira fica explicitada.

"É do conhecimento geral a prática do Lian Gong, mas como ela é muito restrita a gente não tem feito a divulgação não" (GF Gerentes).

"Eu acho que a divulgação do Lian Gong tem que ser ampliada [...] para que os profissionais médicos que atendem os pacientes saibam que existe e podem encaminhar os pacientes". (GF, Gerentes)

Os próprios instrutores em seus grupos focais também comentaram a falta de divulgação por parte dos colegas que trabalham nos Centros de Saúde como na fala:

"Outra equipe que também não apoia, [...] é os meninos do "posso ajudar", entra e sai meninos do "posso ajudar" e eles não sabem nem o que é o Lian Gong" (GF, Instrutores).

Além de divulgação nos Centros de Saúde, faltam inclusive estudos que avaliem a sua efetividade quanto práticas de Promoção da Saúde ${ }^{(18)}$. Muito embora a Política Nacional de Práticas Integrativas e Complementares tenha incentivado algum crescimento das PIC no SUS é evidente a sua marginalização dentro do contexto da Atenção Primária à Saúde ${ }^{(5)}$.

Outro ponto evidente no relato dos profissionais é a dificuldade de aceitação, pelos profissionais inseridos nas equipes, da prática como uma das rotinas de trabalho, por entenderem que o Lian Gong não se configura como procedimento reconhecido como assistencial-curativista.

A visão limitada da saúde dos indivíduos utilizada por algumas organizações fica caracterizada uma visão biológica reducionista, abrindo espaço para a discussão dos profissionais gestores, trabalhadores, pesquisadores e usuários para o desenvolvimento de modelos alternativos que favoreçam os arranjos do sistema de saúde de maneira mais integral e eficiente ${ }^{(19)}$. 
Atualmente, percebem-se no Brasil diversas formas de arranjos nos serviços de saúde, sendo comum referir aos tipos de modelos assistenciais no Sistema Único de Saúde ou na saúde suplementar que a organização dos sistemas de saúde é ainda intensamente voltada aos médicos e hospitais ${ }^{(19)}$. Em geral, os profissionais da saúde se voltam para o tratamento ou assistência de agravos e não consideram o contexto em que o usuário está inserido, sendo as intervenções embasadas, ainda nos conceitos do modelo biomédico e no adoecimento ${ }^{(20)}$.

A edificação de um modelo de atenção à saúde capaz de manter relações com os usuários, trabalhadores e gestores, não é uma tarefa fácil e, para se conseguir efetivar um novo modelo, necessita-se de arranjos bem estruturados ${ }^{(14)}$.

As PIC podem ser consideradas como estratégias de revitalização do sistema de saúde e de mudanças no padrão biologizante e medicalizante do cuidado e da Promoção da Saúde(21).

"[...] você não vê motivação de todas as equipes, é claro que tem uma equipe ou outra que te encaminham, nem todas as equipes abraçam a causa, não participam, então eu acho que é esse apoio formal mesmo" (GF, INSTRUTORES).

A educação permanente é capaz ampliar os saberes e práticas dos profissionais, antes moldados pelas limitações encontradas no modelo biomédico ${ }^{(22)}$. A busca por alternativas que possam contribuir para a efetivação das ações de enfrentamento das barreiras dentro da atenção primária deve priorizar o apoio institucional e a educação permanente dos profissionais.

Em relação ao acesso, os relatos reforçam que existem dois aspectos a se considerarem. A dificuldade de se ter acesso aos locais de prática e o acesso a diferentes faixas etárias de praticantes. $\mathrm{O}$ acesso é a possibilidade que o usuário tem de chegar ao local de assistência e obter o serviço e compreende na facilidade em ter acesso ao local da prática (à distância e o tempo necessário para chegar), a conveniência dos horários, o conforto do ambiente e também a aceitabilidade quanto à satisfação do usuário ${ }^{(23)}$. Foi verificado que o acesso representa uma barreira para a adesão dos praticantes conforme as seguintes falas:

"[...] é um morro bem grande e os idosos não conseguiram subir o morro pra poder praticar e tem dificuldade" (GF, GERENTES).

No estudo a respeito do perfil dos praticantes de LG18T em Belo Horizonte fica claro que o perfil dos praticantes é composto quase que em sua totalidade por mulheres, idosas, aposentadas ou donas de casa, revelando que ainda existe uma falta de acesso para outros públicos, seja pela inconveniência dos horários ou por outros $\operatorname{motivos}^{(5)}$.
No estudo feito sobre a inserção do Lian Gong na Estratégia Saúde da Família de Samambaia no Distrito Federal dentre as dificuldades elencadas como fatores de desistência dos usuários na prática, a necessidade de capacitação de um número maior de profissionais, a oferta em outros horários e outros locais foram as mais ressaltadas $^{(24)}$. A fala da gerente de um Centro de Saúde de Belo Horizonte mostra esta barreira:

"Então o periodo dela de férias ela já está assim, um pouco chorosa, ai como que eu vou fazer com o Lian Gong? Porque não tem substituto pra gente, então este é um fator que dificulta a pessoa da retorno pro profissional e é um dificultador" (GF, NOROESTE, GERENTES).

O baixo número de trabalhadores capacitados como instrutores de LG18T não estão conduzindo a prática corporal por falta de apoio gerencial e da própria equipe local; sobrecarga de agenda; não priorização da ação de Promoção da Saúde; transferência de local de trabalho e remanejamento para cumprimento de outras tarefas, principalmente por muitos instrutores ocuparem cargos de gerente de Centros de Saúde.

A oferta de institutos que formam profissionais de saúde para atuarem nas Práticas Integrativas e Complementares (PIC) e chegaram à conclusão que para efetivar e ampliar a implementação das PIC no Brasil é preciso não só incrementar o número de instituições que ofereça cursos e especializações é importante também que eles estejam alinhados com as propostas, princípios e diretrizes do SUS, que exista um estímulo para a realização destes cursos e que seja facilitada a capacitação destes ${ }^{(21)}$.

Apesar da PNPIC aprovada pela portaria 971 de 2006 do Ministério da Saúde afirmar que dará apoio técnico e financeiro aos projetos de qualificação dos profissionais que atuem na estratégia Saúde da Família, ainda existe um número baixo de instrutores de Lian Gong que contemple a demanda da Atenção Primária no município de Belo Horizonte.

\section{CONSIDERAÇÕES FINAIS}

Apesar de o Lian Gong estar inserido no município de Belo Horizonte desde o ano de 2007 e apresentar atualmente, cerca de 15.000 praticantes, o reconhecimento desta prática corporal como estratégia de promoção da saúde ainda se constitui como um desafio institucional.

A categoria identificada, nesse estudo, aponta para a periferização das práticas integrativas no modelo assistencial, que é reforçada pela cultura institucional, pois ainda está enraizada no modelo curativista, o qual não valoriza as práticas que possam contribuir no aumento do gradiente de saúde da população, vinculada ao Sistema Único de Saúde. 
Nesse sentido, ao privilegiar práticas de promoção da saúde na atenção primária, torna-se possível o acesso da população à melhoria de sua condição de saúde. Nesse aspecto, essas estratégias podem sinalizar uma possível inversão de modelo assistencial, onde a população reconhece os serviços de saúde, como um locus produtor de ações, voltadas para o bem estar em detrimento da assistência exclusiva ao adoecimento.

\section{REFERÊNCIAS}

1. Ministério da Saúde (Br). Portaria $n^{\circ} 971$, de 03 de maio de 2006. Aprova a Política nacional de práticas integrativas e complementares no SUS - PNPIC-SU. Diário Oficial da União. 03 Mai 2006.

2. Moretti AC, Almeida V, Westphal MF, Bógus CM. Práticas Corporais/Atividade Física e Políticas Públicas de Promoção da Saúde. Saúde Soc. 2009; 18(2): 346354.

3. Organização Mundial da Saúde (OMS). Tradicional Medicine Strategy; 2014.

4. Cintra MER, Pereira PPG. Body perceptions identified among traditional Chinese medicine patients and professionals from the Academic Health Center of Butantã. Saúde Soc. 2012; 21(1): 193-205.

5. Tesser CD, Souza IMC. Atenção Primária, Atenção Psicossocial, Práticas Integrativas e Complementares e suas afinidades eletivas. Saúde Soc. 2012; 21(2): 336350 .

6. Luz MT, Cultura Contemporânea e medicinas alternativas: novos paradigmas em saúde no fim do século XX. Physis Revista de Saúde Coletiva. 2005; 15(sup.):145-176.

7. Lee ML. Lian Gong em 18 Terapias - Forjando um corpo saudável. São Paulo: Pensamento; 1997.

8. Livramento G, Franco T, Livramento A. A ginástica terapêutica e preventiva chinesa Lian Gong/Qi Gong como um dos instrumentos na prevenção e reabilitação da LER/DORT. Rev. Bras. Saúde Ocupacional. 2010; 35(121):74-86.

9. Rodrigues, SC, Philomeno BLS, Bahia RR, Abreu MNS, Campos KFC, Roquete FF, et al. Benefícios da prática de Lian Gong em 18 Terapias no município de Belo Horizonte Minas Gerais. In: Anais IV Congresso Online - Gestão, Educação e Promoção da Saúde; 2015 Nov 10-14; online; 2015.

10. Contatore AO, Barros NF, Durval MR, Barrio PCCC, Coutinho BD, Santos JA, et al. The use, care and policy of complementary and integrative practices in primary health care. Ciênc Saúde Coletiva. 2015;20(10): 326373.

11. Minayo MCC. O desafio do conhecimento: pesquisa qualitativa em saúde. São Paulo, Rio de Janeiro: Hucitec, Abrasco; 2010.

12. Bardin L. Análise de Conteúdo. Lisboa: Edições 70; 2009.

13. Randow R, Campos KFC, Roquete FF, Silva LTH, Duarte VES, Guerra VA. Ações de Promoção da Saúde na Atenção Primária à Saúde: o Programa de Lian Gong em 18 Terapias de Belo Horizonte, Minas Gerais, Brasil. In: 5 Congresso Ibero-Americano em Investigação Qualitativa (CIAIQ2016); 2016 Jul 1214; Porto; 2016.

14. Casanova AO, Teixeira MB, Montenegro E. O apoio institucional como pilar na cogestão da atenção primária à saúde: a experiência do Programa TEIAS - Escola Manguinhos no Rio de Janeiro, Brasil. Ciênc Saúde Coletiva. 2014;19(11):4417-26.

15. Rodrigues CC, Ribeiro KSQS. Promoção da Saúde: a concepção dos profissionais de uma unidade de Saúde da Família. Trab. Educ. Saúde. 2012; 10(2): 235-255

16. Ministério da Saúde (Br). Portaria $n^{\circ} 2.446$, de 11 de novembro de 2014. Redefine a Política nacional de Promoção da Saúde- PNPS-SUS. Diário Oficial da União. 11 Nov 2014.

17. Yamakawa HHA, Bugulin E, Campos L, Tavares D. A Interface das Doenças Crônicas e a Prática do Lian Gong. Projeto: DANT - Doenças e Agravos Não Transmissíveis. Supervisão Técnica de Saúde Penha. São Paulo; 2006.

18. Westphal MF. Concepções e abordagens na avaliação em promoção da saúde. Dilemas da avaliação em promoção da saúde: como orientar a produção científica brasileira. Ciência e Saúde Coletiva. 2004; 9(3):534536.

19. Faria OP, Coelho IB, Werneck AZF, Santos MA. Modelos Assistenciais e a atenção básica à saúde. Belo Horizonte: Editora Coopmed; 2010.

20. Lazzarotti Filho A, Silva AM, Antunes PC, Silva APS, Leite JO. O termo práticas corporais na literatura científica brasileira e sua repercussão no campo da Educação Física. Movimento. 2010; 16(1): 65-84.

21. Azevedo E, Pelicioni MCF. Práticas integrativas e complementares de desafios para a educação. Trabalho, Educação e Saúde. 2011; 9(3): 361-78. 
22. Buss PM, Pellegrini FA. A saúde e seus determinantes sociais. Physis. 2007;17(1):77-93.

23. Shimazaki ME. A Atenção Primária à Saúde. In: MINAS GERAIS. Escola de Saúde Pública do Estado de Minas Gerais. Implantação do Plano Diretor da Atenção Primária à Saúde. Oficina 2 e 3. 1- Análise da atenção primária à saúde. Guia do participante. Belo Horizonte: ESPMG; 2009.

24. Andrade SC, Leão DAO, Silva KV, Melo BC, Guimarães MAS, Paulo GP. Experiência da inserção do Lian Gong na estratégia saúde da família de Samambaia - Distrito Federal. Com. Ciências Saúde. 2013; 22(4): 9-18.

\section{Endereço primeiro autor:}

Raquel Randow

Escola de Enfermagem da Universidade Federal de Minas Gerais

Avenida Professor Alfredo Balena, 190

Bairro: Santa Efigênia

CEP: 30130-100 - Belo Horizonte - MG - Brasil

E-mail: raquelrondow@gmail.com

\section{Endereço para correspondência:}

Vanessa de Almeida Guerra

Escola de Enfermagem da Universidade Federal de Minas Gerais

Avenida Professor Alfredo Balena, 190

Bairro: Santa Efigênia

CEP: 30130-100 - Belo Horizonte - MG - Brasil

E-mail: vanessaalmeidaufmg@gmail.com 